\title{
The efficacy and safety of moderate aerobic exercise for patients with Parkinson's disease: a systematic review and meta-analysis of randomized controlled trials
}

\author{
Yang $\mathrm{Li}^{1}$, Hongling Song ${ }^{2}$, Lihua Shen ${ }^{3}$, Yanan Wang ${ }^{4}$ \\ ${ }^{1}$ Medical School (School of Nursing), Nantong University, Nantong, China; ${ }^{2}$ Department of Geriatric Rehabilitation, Affiliated Hospital of Nantong \\ University, Nantong, China; ${ }^{3}$ Affiliated Hospital of Nantong University, Nantong, China; ${ }^{4}$ Shanghai Fifth People's Hospital, Fudan University, \\ Shanghai, China \\ Contributions: (I) Conception and design: Y Li, H Song; (II) Administrative support: H Song, L Shen; (III) Provision of study materials or patients: Y \\ $\mathrm{Li}$; (IV) Collection and assembly of data: Y Li, Y Wang; (V) Data analysis and interpretation: Y Li, Y Wang; (VI) Manuscript writing: All authors; (VII) \\ Final approval of manuscript: All authors. \\ Correspondence to: Hongling Song. Department of Geriatric Rehabilitation, Affiliated Hospital of Nantong University, Nantong 226000, China. \\ Email: jsyjsong@sina.com.
}

Background: Exercise therapy is an important component of non-drug treatment for Parkinson's disease (PD). However, the impact of moderate aerobic exercise on PD remains unclear. The purpose of this systematic review was to evaluate the efficacy and safety of moderate aerobic exercise for patients with PD.

Methods: Databases including the Cochrane Library, PubMed, Web of Science, EMBASE, Chinese Biomedical Literature (CBM) Database, Chinese National Knowledge Infrastructure (CNKI), WanFang, and the Weipu Database for Chinese Technical Periodicals (VIP) were searched electronically from the date of inception of the database to June 2020 to recruit relevant randomized controlled trials (RCTs) investigating the efficacy and safety of moderate aerobic exercise on balance and other symptoms in patients with PD. Literature screening, data extraction, and quality evaluation were carried out. Revman5.1 (http:// ims.cochrane.org/revman) was used for data analysis.

Results: In total, nine RCTs with 444 patients were included in this study. Most of the included trials had a low risk of bias and high methodological quality. The meta-analysis suggested that moderate aerobic exercise is effective in improving balance [weighted mean difference (WMD) $=-0.42,95 \%$ confidence interval (CI): -0.59 to $-0.25, \mathrm{P}<0.001]$ and gait $(\mathrm{WMD}=49.97,95 \% \mathrm{CI}: 17.84-82.10, \mathrm{P}<0.001)$ in patients with $\mathrm{PD}$, but not in motor symptoms (WMD $=-2.14,95 \% \mathrm{CI}:-4.86-0.58, \mathrm{P}=0.12$ ). Subgroup analysis showed that the improvement in the quality of life of PD patients was affected by different types of exercise, and the treadmill subgroup improved significantly (WMD =-3.90, 95\% CI: -5.02 to -2.78 ), $\mathrm{P}<0.001$ ).

Conclusions: On the whole, moderate aerobic exercise effectively improves balance and gait in patients with PD, but the effect on motor symptoms is not obvious. Different exercise styles have varying effects on the quality of life of PD patients. It is necessary to standardize the exercise program further and carry out indepth research in the future.

Keywords: Parkinson disease; aerobic exercise; balance; gait; quality of life; meta-analysis

Submitted Aug 21, 2020. Accepted for publication Dec 03, 2020.

doi: 10.21037/apm-20-1661

View this article at: http://dx.doi.org/10.21037/apm-20-1661 


\section{Introduction}

Parkinson's disease (PD) is a common progressive neurodegenerative disease characterized by motor symptoms such as tremor, bradykinesia, postural instability, and gait disturbances (1). In China, the prevalence rate of PD in older adults (over 65 years) is 1.7\%, accounting for about one-third of the total patients worldwide, and is increasing at a rate of approximately 100,000 per year (2). With the rapid growth of the elderly population in China, it is estimated that the number of PD patients will reach 4.94 million by 2030. PD is one of the "three killers" of Chinese life expectancy (together with cardiocerebrovascular diseases and cancer), which seriously affects the quality of life of patients and significantly exacerbates the social and economic burden $(3,4)$. At present, drug therapy is one of the main means of clinical treatment for PD patients but is mired by issues of low efficacy and high adverse event frequency (5). Studies in China and across the world have found that various non-pharmacological treatment methods can significantly affect both the motor and non-motor symptoms of PD (6). Among these methods, exercise therapy can strengthen the balance and gait ability of PD patients, improve negative emotions, and enhance the quality of life (7-9). It also offers further advantages including being efficient, economical, and accessible, and has been widely used in clinical, community, and auxiliary treatment.

Aerobic exercise refers to physical exercise. The whole body's main muscle groups participate in the increase of heart rate under conditions of sufficient oxygen supply and can include activities like treadmill running, jogging, bicycle training, and other exercise. It is the most common exercise intervention method for PD patients and improves their cardiopulmonary endurance and exercise ability $(10,11)$. Studies have shown that increased exercise intensity can accelerate the motor cortex's nerve activity (12), but high-intensity exercise is close to the anaerobic threshold, which will increase the risk of injury and chronic inflammation (13). Given that PD is mainly characterized by motor symptoms and the safety of intervention, combined with information from the guidelines issued by the World Health Organization (WHO) and the American College of Sports Medicine (ACSM) for healthy people $(14,15)$, it is believed that moderate-intensity aerobic exercise [that is, where the heart rate maintained at $64-76 \%$ of the maximum heart rate reserve (HRR)] can achieve the best exercise effect and has good adaptability (16).
Numerous investigations have focused on the effectiveness of high-intensity or low-intensity aerobic exercise on PD $(17,18)$, but have neglected moderateintensity exercise effectiveness, despite it being recommended by several guidelines (19). Meanwhile, different studies have contrasting views on whether moderate-intensity aerobic exercise can improve PD symptoms $(20,21)$. There is presently a lack of accurate information and quantitative analysis (such as systematic evaluation), which has affected the development of evidence-based rehabilitation for PD.

Therefore, the present study explored the effects of moderate-intensity aerobic exercise in PD patients to provide an evidence-based foundation for the design of exercise programs and improve clinical practice for PD patients in the future.

We present the following article in accordance with the PRISMA reporting checklist (available at http://dx.doi. org/10.21037/apm-20-1661).

\section{Methods}

\section{Data sources and search strategies}

A literature search of the electronic databases of The Cochrane Library, PubMed, Web of Science, EMBASE, Chinese Biomedical Literature Database (CBM), Chinese National Knowledge Infrastructure (CNKI), WanFang, and the Weipu Database for Chinese Technical Periodicals (VIP) was performed from the date of inception of the database to June 2020. The following keywords and their combinations were used: "Parkinson disease", "Parkinson's disease", "Primary Parkinsonism", "Lewy Body Parkinson's disease", "Idiopathic Parkinson Disease", "PD", "Exercise", "Physical Activity", "Physical Exercise", "Acute Exercise", "Exercise", "Isometric", "Aerobic Exercise", and "Exercise Training". Literature was also identified by citation tracking using reference lists from papers and internet searching. The reference lists of full articles and dissertations were also reviewed.

\section{Selection criteria and exclusion}

The following inclusion criteria was applied to our literature search:

(I) Research type: randomized controlled trials (RCTs);

(II) Participants: subjects were clinically diagnosed with PD; 
(III) Intervention: moderate-intensity aerobic exercise was defined according to the classification standard of the ACSM (15), which refers to exercise where the HRR of subjects is $64-76 \%$; the moderateintensity target heart rate area was set in advance and controlled by heart rate monitor during the intervention; exercise frequency, time, and course of treatment were not limited.

(IV) Comparison: placebo combined with non-aerobic exercise intervention or routine nursing, but not including resistance exercise, balance exercise, Tai Chi or yoga, and other physical and mental activities.

(V) Outcomes: outcomes included at least one of balance, gait, motor symptoms, or health-related quality of life.

(VI) Study design: Each study's purpose and method were the same or similar, and the RCT study must include a moderate aerobic treatment group and a routine control group.

(VII) Other: studies contained available data for the meta-analysis and were published in either English or Chinese.

Studied were excluded for the following reasons:

(I) The effect of a non-aerobic exercise intervention was evaluated (including resistance exercise, balance exercise, Tai Chi, dance, or comprehensive intervention with other sports).

(II) No placebo was used as a control, and no outcome data were provided.

\section{Data extraction}

Two reviewers (YL and YNW) independently extracted data from eligible articles with a standard form. Collected data included the following: first author; publication year; country; study design; inclusion criteria; the frequency, intensity, and duration of the intervention; and the HRR range. The participant's information included the number, age, evaluation status, related outcomes, and adverse effects. Any discrepancies between reviewers were resolved by consensus. The corresponding author was contacted if sufficient data of an eligible study could not be obtained from the full text.

\section{Study quality assessment}

Quality assessment was carried out independently by two reviewers ( $\mathrm{YL}$ and $\mathrm{YNW}$ ) using the Cochrane risk of bias tool (22), which includes seven domains: randomization sequence generation, allocation concealment, blinding of participants, blinding of study personnel, blinding of outcome assessors, incomplete outcome data, selective reporting, and other sources of bias. The corresponding judgments of "low bias", "unknown bias", and "high bias" were made for each study.

\section{Statistical analysis}

The meta-analysis was performed using the Cochrane Collaboration software [Review Manager 5 (RevMan) version 5.1 for Windows. http://ims.cochrane.org/revman]. Mean difference (MD) or standardized mean difference (SMD) was used as the statistical effect quantity, and $95 \%$ confidence intervals (CIs) were calculated by effect analysis. Heterogeneity was evaluated based on the $\mathrm{I}^{2}$ statistic. Studies with $\mathrm{I}^{2}>50 \%$ and $\mathrm{P}<0.05$ were considered as showing high heterogeneity, and the random-effects model was used; otherwise, a fixed-effects model was applied. If the heterogeneity was obvious, subgroup analysis or sensitivity analysis was conducted according to sample baseline and intervention measures to find the source of heterogeneity. The publication bias analysis was assessed by funnel plot. However, the plot could not be completed due to the small number of RCTs included.

\section{Results}

\section{Search results}

Our search strategy yielded a total of 3,143 potentially related references. After removing duplicates, the number of papers was reduced to 1,918 , and we excluded a further 1,884 articles by reading the titles and abstracts. Of the 34 full-text articles assessed for eligibility, 22 were excluded for the following reasons: not an RCT, inconsistent intervention, and no outcomes of interest described. We included nine RCTs that were unanimously agreed on by the two reviewers. Figure 1 displays the flow of the study according to the screening details.

\section{Study characteristics and risk of bias}

Table 1 summarizes the characteristics of the nine included studies. They comprised a total of 444 participants (21,23-30). Of these, 217 participants were in the comparison group 


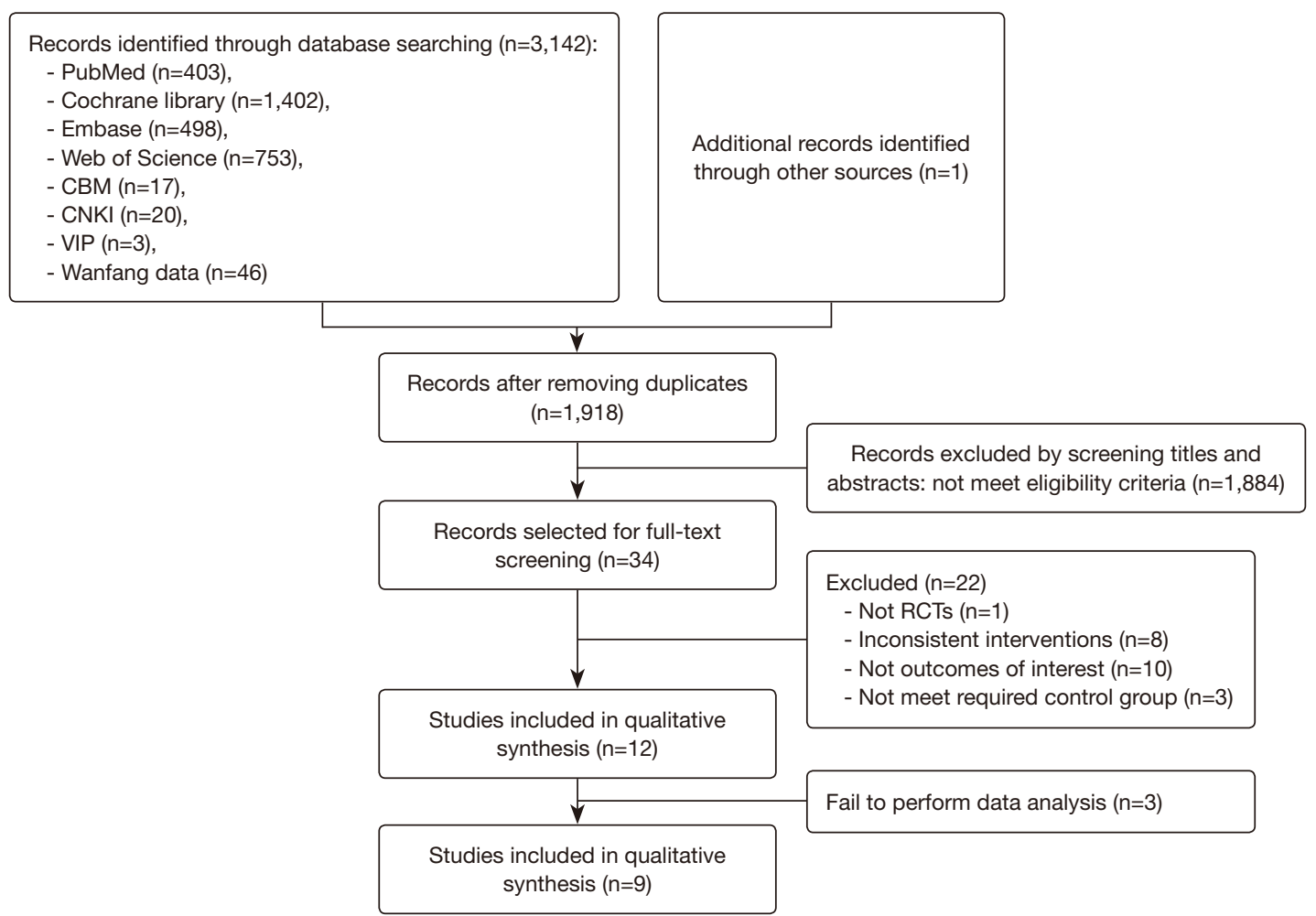

Figure 1 Search results of the study.

(49\%), while 227 were in the moderate aerobic exercise group (51\%). The duration of PD included less than 1 to 8 years. Most trials recruited participants with mild-tomoderate PD, including three $(21,27,28)$ with Hoehn and Yahr stage I to II and five $(23,25,26,29,30)$ with Hoehn and Yahr stage I to III.

All of the eligible studies reported aerobic exercise interventions including treadmill training, bike training, walking, and jogging. The interventions in the control group were simple, and consisted of routine or home-based exercises. The intervention length ranged from 8 weeks to 16 months. Exercise training frequency varied between two and seven sessions per week, with each session lasting between 20 and 90 minutes. The average training intensity was moderate, and the HRR ranged from $50 \%$ to $80 \%$.

The assessment of bias risk is shown in Figures $2 \mathrm{~A}$ and $2 \mathrm{~B}$. Of the nine included articles, seven $(21,23$, $25-28,30)$ described the generation of random sequences, and six $(21,25-28,30)$ used an adequate method of allocation concealment. Six studies $(21,23,25-28)$ reported subject- and therapist-blinding, but only one study report (28) clearly described the double-blind process, which was a common drawback for the non-pharmacological clinical trials. Only three trials $(21,28,30)$ completed an intention-to-treat analysis. For the remaining items on Cochrane's tool, the eligible studies showed low risks and high methodological quality.

\section{Meta-analysis}

\section{The Timed Up and Go Test (TUG)}

Four studies reported changes in the TUG, and the results are shown in Figure 3. There was no significant heterogeneity among the studies $\left(\mathrm{P}<0.00001, \mathrm{I}^{2}=32 \%\right)$, and a fixed-effects model was adopted. Compared with the placebo, moderate-intensity aerobic exercise showed a significant difference in improving balance in patients with $\mathrm{PD}(\mathrm{P}<0.00001, \mathrm{MD}=-0.42,95 \% \mathrm{CI}:-0.59$ to -0.2$)$.

\section{6-Minute Walking Test (6MWT)}

In this meta-analysis, six studies reported the gait data. Due to the high heterogeneity between the two groups, a random effects model was used to analyze this outcome $\left(\mathrm{P}=0.002, \mathrm{I}^{2}=74 \%\right)$. Subgroup analysis was performed based on the duration of intervention, and the results are shown in Figure 4. The heterogeneity of the two groups was 


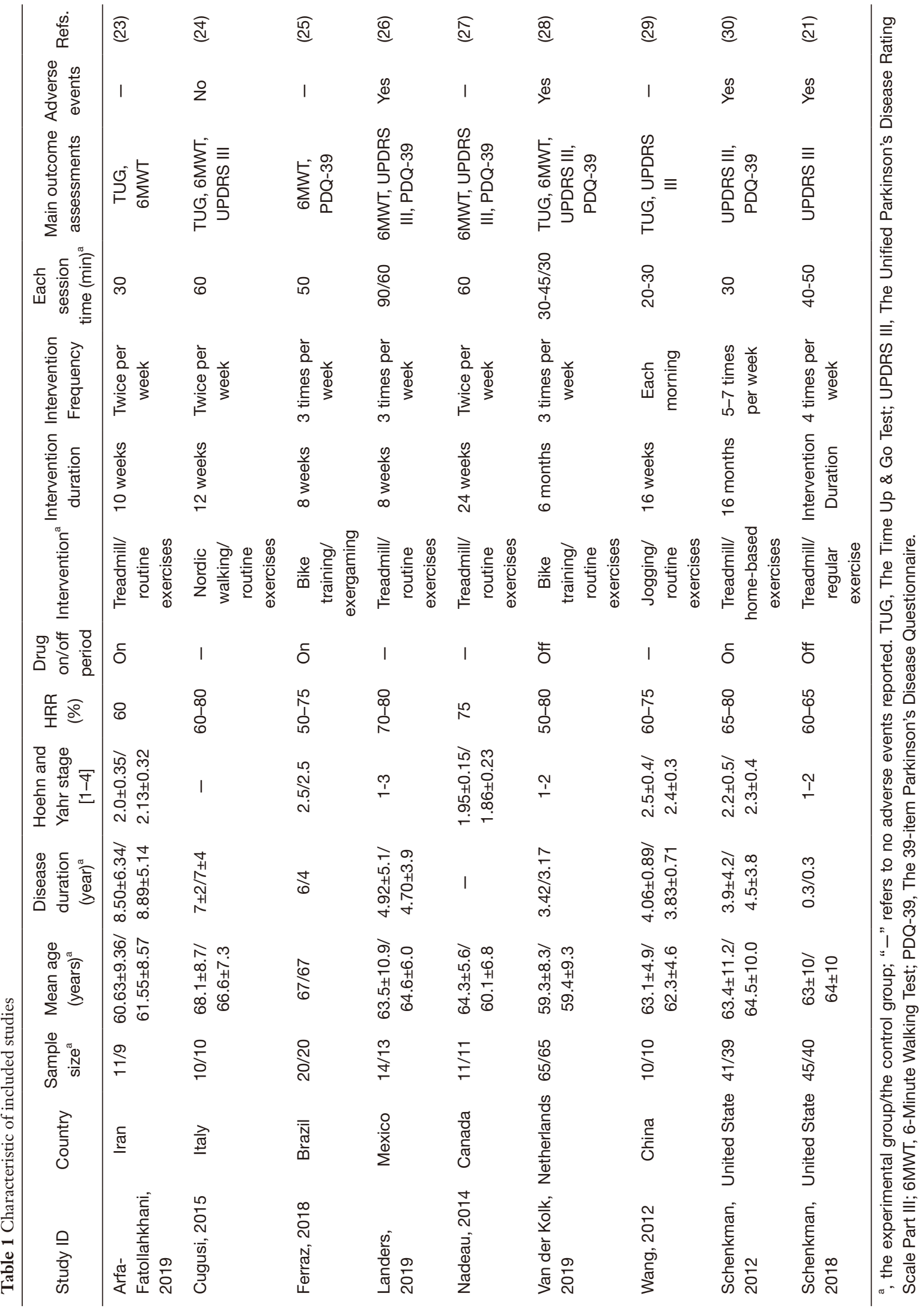



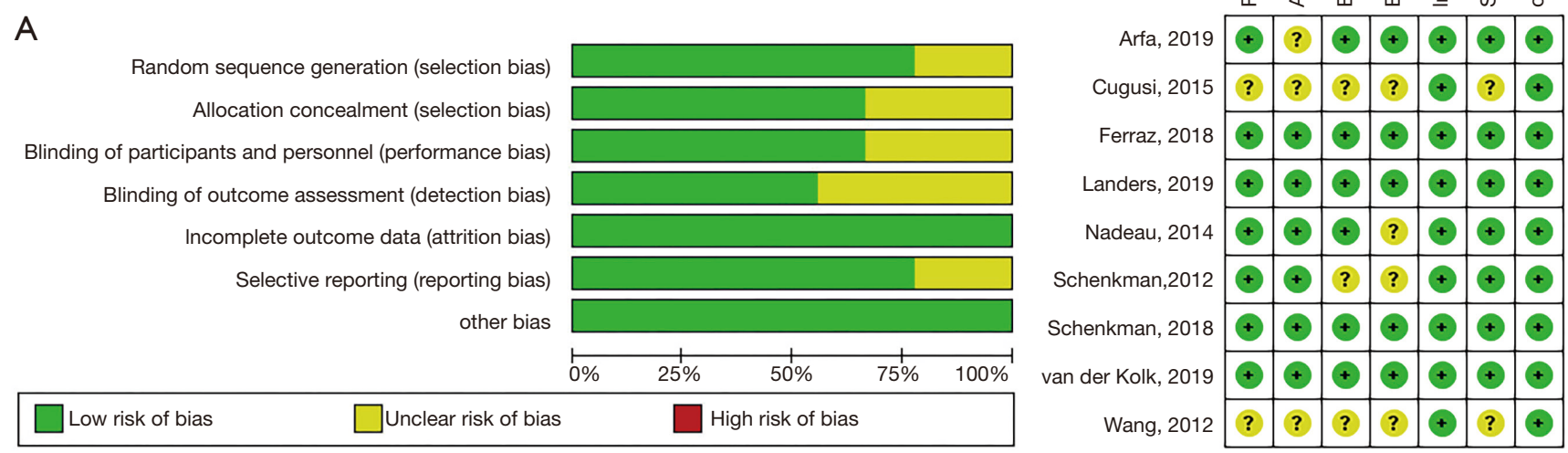

Figure 2 Risk of bias summary of included studies. (A) Risk of bias graph; (B) risk of bias summary.

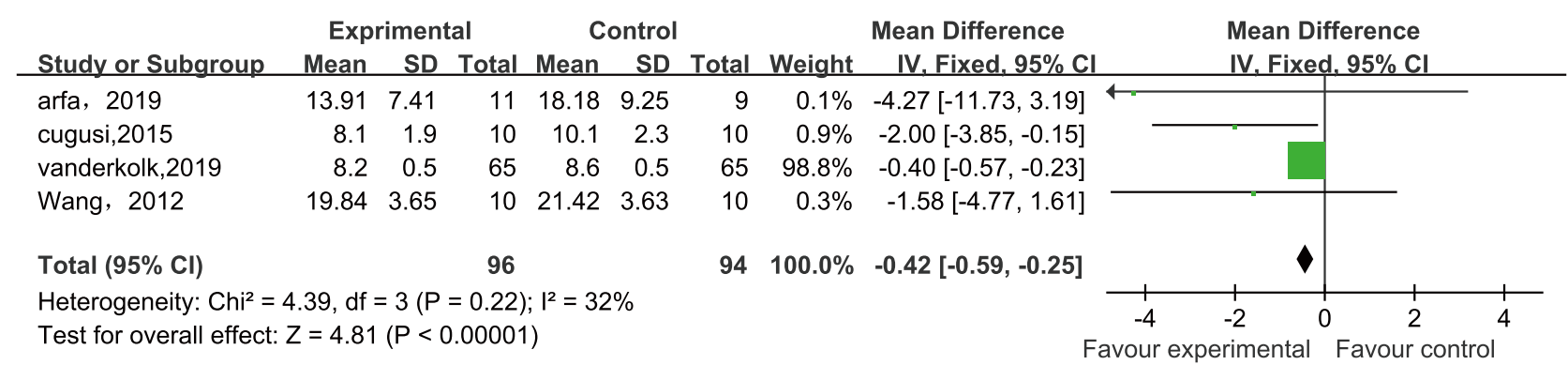

Figure 3 The effect of moderate aerobic exercise on the Timed Up and Go Test (TUG) of Parkinson's disease (PD).

significantly decreased $\left(\mathrm{I}^{2}=0 \%\right)$, regardless of whether the intervention duration was $\leq 12$ or $>12$ weeks, and moderate aerobic exercise showed superior effects in improving gait in both groups.

\section{The Unified Parkinson's Disease Rating Scale Part III (UPDRS III)}

Seven studies reported the data of the UPDRS III (Figure 5). The heterogeneity between the two groups was high
$\left(\mathrm{P}<0.00001, \mathrm{I}^{2}=93 \%\right)$, and a random effects model was used to analyze this data. The aggregated results showed that moderate aerobic exercise was not associated with an improvement in UPDRS III $(\mathrm{P}=0.12, \mathrm{MD}=-2.14,95 \% \mathrm{CI}$ : -4.86-0.58). Subgroup analysis was performed according to the research characteristics that may cause heterogeneity, including intervention time ( $\leq 60$ or $>60$ minutes), duration $(\leq 12$ or $>12$ weeks), and intervention mode (treadmill, bicycle, or jogging). However, the heterogeneity was still 


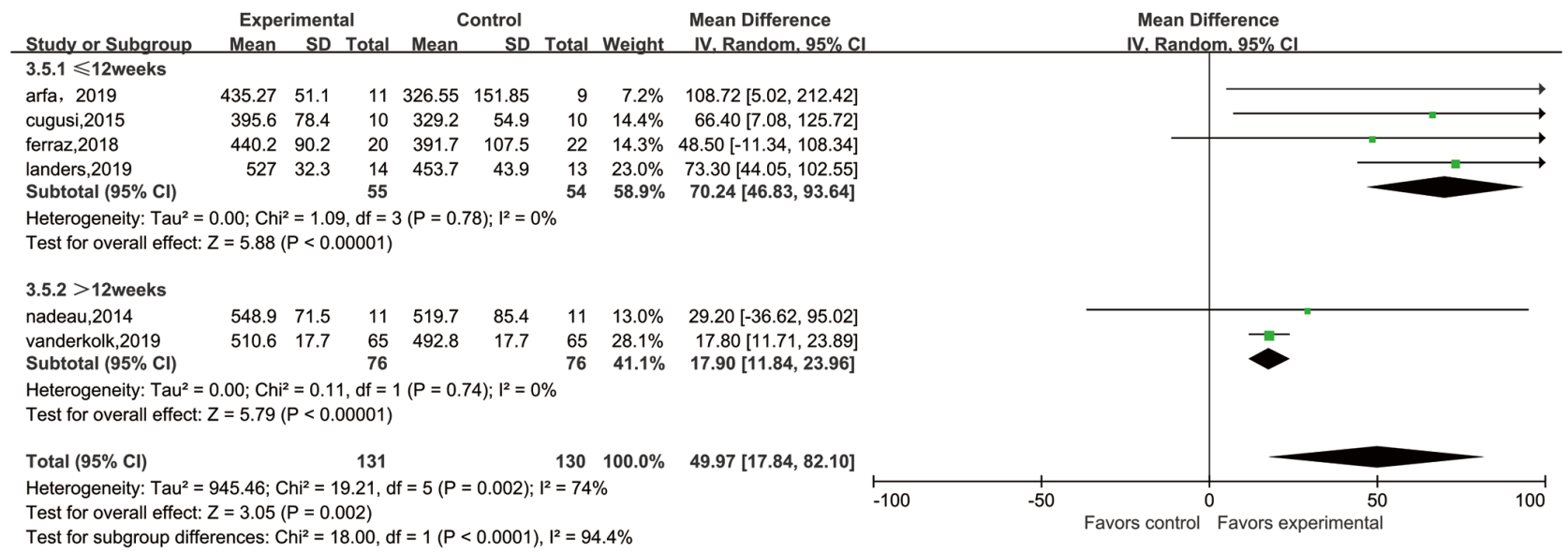

Figure 4 The effect of moderate-intensity exercise on 6-Minute Walking Test (6MWT) of Parkinson's disease (PD).

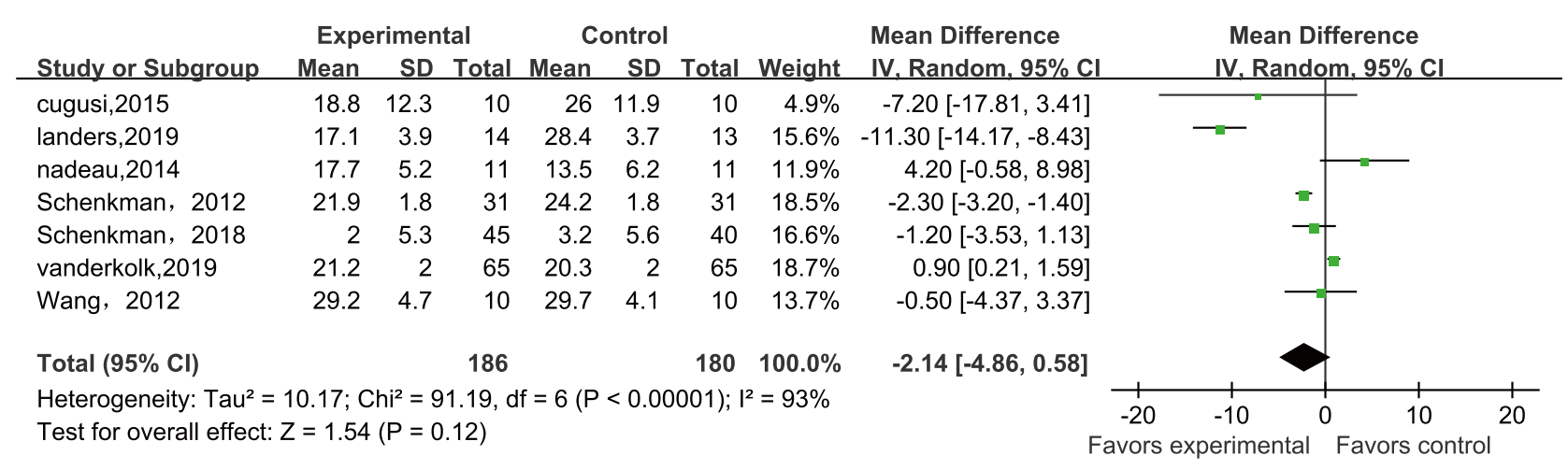

Figure 5 The effect of moderate-intensity exercise on the Unified Parkinson's Disease Rating Scale Part III (UPDRS III) of Parkinson's disease $(\mathrm{PD})$.

high. It is suggested that the exercise pattern cannot explain the source of heterogeneity among the studies, and thus further careful analysis is required (Table 2).

\section{The 39-item Parkinson's Disease Questionnaire (PDQ-39)}

Five studies investigated the changes in the PDQ-39, and of these, the data from one study could not be analyzed. A random effects model was used to analyze these outcomes due to the high heterogeneity $\left(\mathrm{P} \leq 0.00001, \mathrm{I}^{2}=89 \%\right)$. Overall, compared with the placebo, the experimental group's quality of life was not significantly improved $(\mathrm{P}=0.12$, $\mathrm{MD}=-2.60$, 95\% CI: -5.83-0.64). However, subgroup analysis showed that heterogeneity decreased after grouping based on the intervention mode (treadmill, bicycle, or jogging). The score of PDQ-39 in the treadmill subgroup was better than that in the control group $(\mathrm{P}<0.00001)$, while there was no significant difference in the other subgroups (Figure 6).

\section{Adverse events}

Five studies $(21,24,26,28,30)$ reported on the occurrence of adverse events during exercise training. Four of these studies $(21,26,28,30)$ described adverse events occurring in both the experimental group and the control group, including mild adverse events (pain, palpitation, sprain/strain) and serious adverse events (falls, fractures, tachycardia, etc.). However, they were unable to determine whether these were related to the exercise program. Only one study (24) reported no adverse events. 
Table 2 Subgroup analysis of the effect of moderate aerobic exercise on UPDRS III of PD

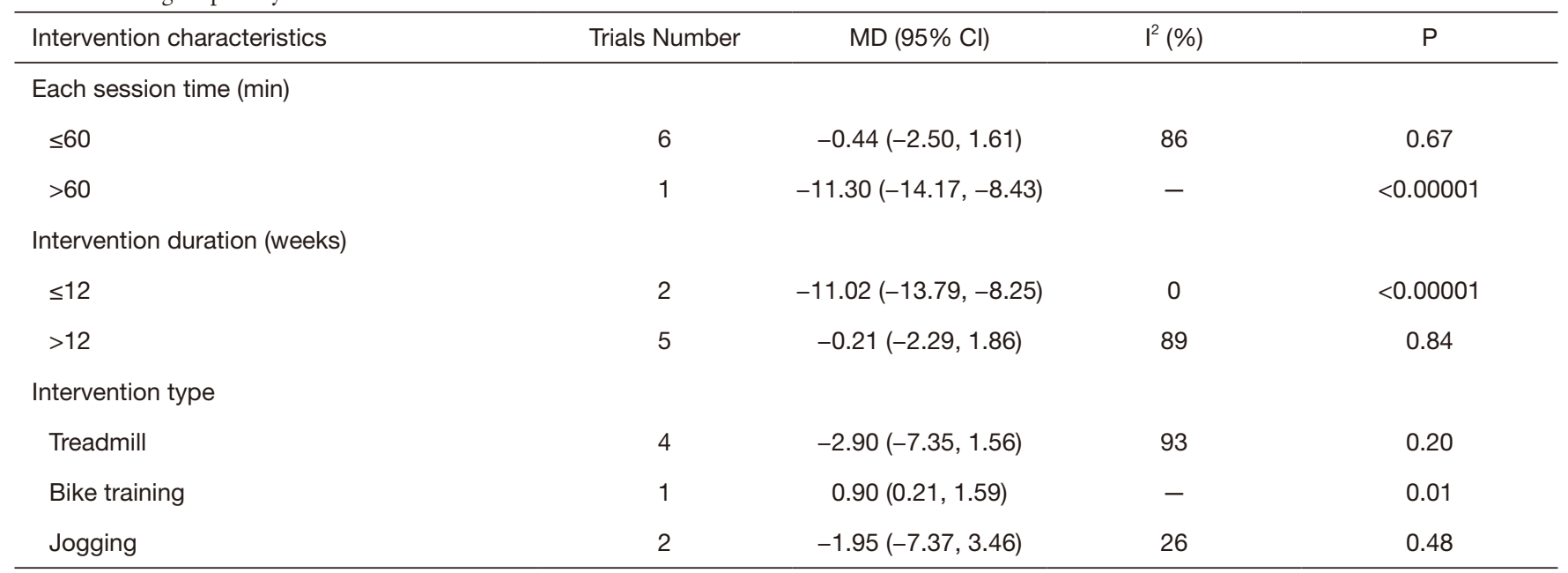

"-" means the heterogeneity analysis cannot be carried out due to the lack of literature included in subgroup analysis. UPDRS III, The Unified Parkinson's Disease Rating Scale Part III; 6MWT, 6-Minute Walking Test; PD, Parkinson's disease.

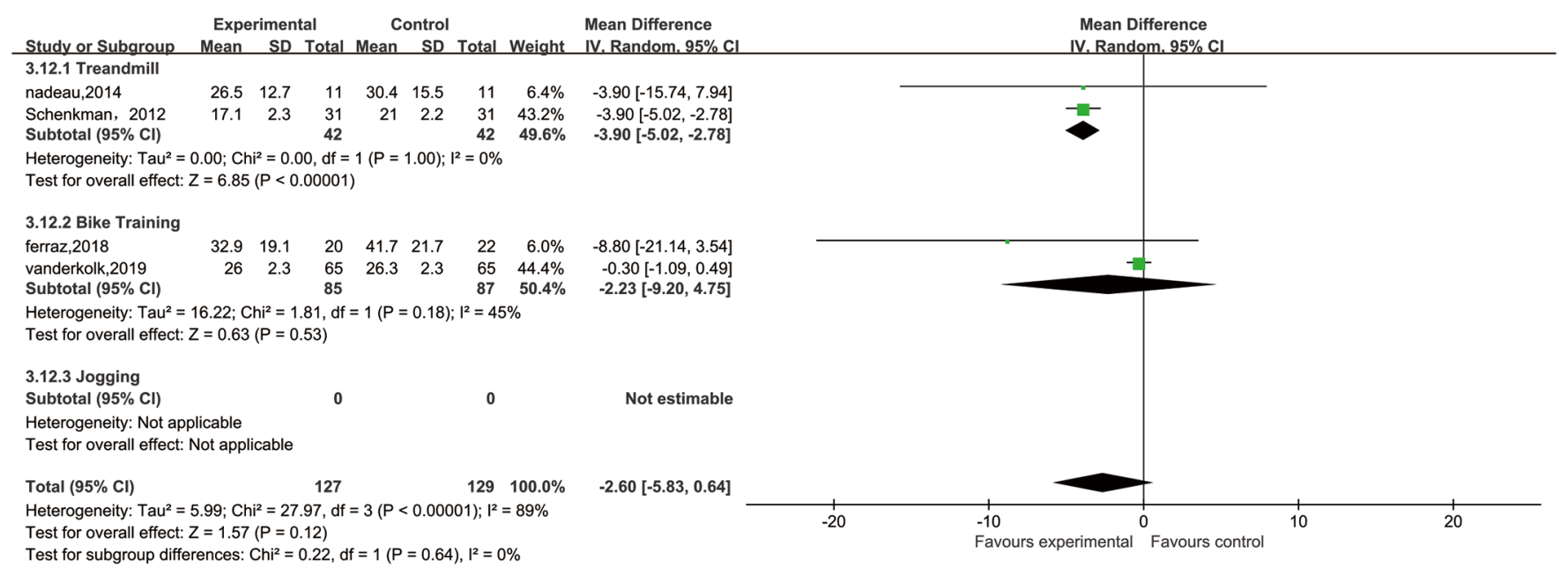

Figure 6 The effect of moderate-intensity exercise on the 39-item Parkinson's Disease Questionnaire (PDQ-39) of Parkinson's disease (PD).

\section{Discussion}

In clinical practice, PD patients with balance disorder and abnormal gait have rapid progress, and the probability of cognitive impairment, falls, and disability is high (31). Consistent with the results of Shen et al. (32) and Fasano et al. (16), this study shows that moderate-intensity aerobic exercise can improve the balance and gait ability of PD patients. Relevant basic experiments show that the level of brain-derived neurotrophic factor (BDNF) is decreased in PD patients' brains, and dopamine neurons are reduced. Aerobic training can induce increased levels of BDNF, protect dopaminergic neurons, participate in the activation and recovery of motor function (33). Zoladz et al. (20) also confirmed that moderate-intensity aerobic exercise lasting 60 minutes and occurring three times a week can achieve therapeutic effect by increasing BDNF levels. Some studies have also shown that aerobic exercise is related to hippocampal volume (34). Walking for more than 40 minutes per week with HRR reaching $60-75 \%$ can significantly delay hippocampal atrophy and relieve disease symptoms.

However, there is still a lack of systematic and standardized exercise programs, and the randomized 
controlled studies based on maximum HRR to determine exercise intensity are very limited. Several small-sample RCTs have drawn different conclusions. Compared with high-intensity exercise (HRR $=70-80 \%)$, low-intensity exercise $(H R R=40-50 \%)$ can significantly improve PD patients' gait. High-intensity aerobic exercise also significantly affects the lower limb activity and exercise symptoms of PD patients (35-37). Taking into account the differences of HRR reference intervals, the disease characteristics of $\mathrm{PD}$, and the safety of intervention, this study further confirmed the effectiveness of moderateintensity aerobic exercise on the exercise ability of PD patients based on the standard of moderate-intensity aerobic exercise recommended by the WHO and ACSM $(14,15)$. Despite this, concerning the effect of different exercise intensities on PD, it is still necessary to further explore the development and promotion of an exercise intensity standard based on the theoretical mechanism and objective indicators.

Also, following subgroup analysis of exercise intervention duration, it was found that the heterogeneity of the gait index decreased significantly, suggesting that variability in intervention duration was the main source of heterogeneity. However, despite differences in the duration of intervention (8 weeks to 16 months), we still found that moderateintensity aerobic exercise positively affected the gait of PD patients, and the short- and long-term intervention benefits may need to be further elucidated by follow-up studies. Meanwhile, the effect of exercise often depends on the frequency, time, and type of exercise. The guidelines issued by the U.S. Department of Health and Human Services (38) suggest that moderate-intensity physical activity should include aerobic exercise for more than 5 days a week, with each session for 30-60 minutes. However, there are considerable differences in the duration of exercise in the included studies, and the results of the interactions between various factors still need to be carefully explained, further explored, and verified.

UPDRS III, which was developed by the International Parkinson and Movement Disorder Society (MDS), is the most widely used clinical assessment scale of motor symptoms for PD, the results of this study suggest that moderate-intensity aerobic exercise has no significant effect on the improvement of exercise symptoms of PD patients with UPDRS III. Also, there is a high heterogeneity and a lack of consistency among the relevant studies $(28,39)$. This may be that UPDRS III is primarily used to evaluate the gait, tremor, and other motor symptoms of PD patients, to help determine the duration and stage of the disease. However, the baseline of the duration and stage of the studies included in this meta-analysis were not homogeneous. Furthermore, it was unclear whether the scale lay in the period of PD drug action (that is, the "on" and "off" period), while some of the included studies did not mention the drugs used. Considering the wide application of the scale, more attention should be paid to data collection and future research analysis.

Gait disorders, postural instability, and other motor symptoms, as the main symptoms of $\mathrm{PD}$, are the key causes of patients' reduced quality of life (31). PDQ-39 is currently recognized as the most comprehensive and widely used specific scale to evaluate PD patients' quality of life. All of the studies included in this meta-analysis used this scale to evaluate the quality of life. The results showed that moderate-intensity aerobic exercise did not significantly improve PD patients' quality of life, which was consistent with the results of Fasano et al. (16). However, heterogeneity analysis further indicated that the treadmill subgroup's quality of life was significantly improved, while the heterogeneity was reduced. Mehrholz et al. (40) also drew similar conclusions. Different exercise styles and types will affect the improvement of quality of life. The reason for this may be related to the varying requirements of different exercise types for patients. Bicycle and other sports have higher technical requirements than the treadmill, which way produce different results. Also, the quality of life of PD patients is affected by motor symptoms and the interaction of emotion, sleep, daily living ability, and other factors, and is difficult to improve in a short period significantly. Aerobic exercise promotes synaptic plasticity and increases the level of non-dopamine transmitters, which can improve sleep, emotion, cognition, and other non-motor symptoms of patients (41). However, few studies have focused on the effect of moderate-intensity aerobic exercise on PD's nonmotor symptoms.

This systematic review also had several limitations that should be noted. Firstly, only Chinese and English language literature was included in this study, which might have produced a publication bias. Secondly, some indicators could not be further consolidated and analyzed due to different evaluation tools. Lastly, although subgroup analysis reduced the heterogeneity of some results, the differences in sample characteristics, exercise intervention programs, and the definition of the threshold value of indicators might still have led to heterogeneity to some extent. 


\section{Conclusions}

This meta-analysis shows that moderate-intensity aerobic exercise based on HRR can effectively improve the balance and gait ability of PD patients. However, the curative effect on the motor symptoms of PD was not significant. Also, different exercise types may differentially affect the improvement of PD patients' quality of life with moderateintensity aerobic exercise, which warrants further exploration. Due to the small number of studies involved in several outcome indicators, the small sample size, and the differences in exercise intervention methods and frequency, future research should expand the sample size, clarify the standard definition, and standardize the exercise program. Although the included studies did not identify significant adverse events of moderate-intensity aerobic exercise, the safety and longterm efficacy still need to be supported by sufficiently reliable evidence before this method can be applied and promoted in clinical and community exercise therapy.

\section{Acknowledgments}

Funding: This study was supported by the Nantong Science and Technology Project of China (No. MS12018044).

\section{Footnote}

Reporting Checklist: The authors have completed the PRISMA reporting checklist. Available at http://dx.doi. org/10.21037/apm-20-1661

Conflicts of Interest: All authors have completed the ICMJE uniform disclosure form (available at http://dx.doi. org/10.21037/apm-20-1661). The authors have no other conflicts of interest to declare.

Ethical Statement: The authors are accountable for all aspects of the work in ensuring that questions related to the accuracy or integrity of any part of the work are appropriately investigated and resolved.

Open Access Statement: This is an Open Access article distributed in accordance with the Creative Commons Attribution-NonCommercial-NoDerivs 4.0 International License (CC BY-NC-ND 4.0), which permits the noncommercial replication and distribution of the article with the strict proviso that no changes or edits are made and the original work is properly cited (including links to both the formal publication through the relevant DOI and the license). See: https://creativecommons.org/licenses/by-nc-nd/4.0/.

\section{References}

1. GBD 2015 Neurological Disorders Collaborator Group. Global, regional, and national burden of neurological disorders during 1990-2015: a systematic analysis for the Global Burden of Disease Study 2015. Lancet Neurol 2017;16:877-97.

2. Li G, Ma J, Cui S, et al. Parkinson's disease in China: a forty-year growing track of bedside work. Transl Neurodegener 2019;8:22.

3. Ma CL, Su L, Xie JJ, et al. The prevalence and incidence of Parkinson's disease in China: a systematic review and meta-analysis. J Neural Transm (Vienna) 2014;121:123-34.

4. Dorsey ER, Bloem BR. The Parkinson Pandemic-A Call to Action. JAMA Neurol 2018;75:9-10.

5. Khan AU, Akram M, Daniyal M, et al. Awareness and current knowledge of Parkinson's disease: a neurodegenerative disorder. Int J Neurosci 2019;129:55-93.

6. Obeso JA, Stamelou M, Goetz CG, et al. Past, present, and future of Parkinson's disease: A special essay on the 200th Anniversary of the Shaking Palsy. Mov Disord 2017;32:1264-310.

7. Miller KJ, Suárez-Iglesias D, Seijo-Martínez M, et al. Physiotherapy for freezing of gait in Parkinson's disease: a systematic review and meta-analysis. Rev Neurol 2020;70:161-70.

8. Dauwan M, Begemann MJH, Slot MIE, et al. Physical exercise improves quality of life, depressive symptoms, and cognition across chronic brain disorders: a transdiagnostic systematic review and meta-analysis of randomized controlled trials. J Neurol 2021;268:1222-46.

9. Tang L, Fang Y, Yin J. The effects of exercise interventions on Parkinson's disease: A Bayesian network meta-analysis. J Clin Neurosci 2019;70:47-54.

10. Silva CM, Travessa AM, Bouça-Machado R, et al. Reporting and methodological quality of clinical trials on exercise therapy for Parkinson's disease. Parkinsonism Relat Disord 2019;69:150-6.

11. Lamotte G, Rafferty MR, Prodoehl J, et al. Effects of endurance exercise training on the motor and non-motor features of Parkinson's disease: a review. J Parkinsons Dis 2015;5:21-41.

12. Takehara N, Tsubaki A, Yamazaki Y, et al. Changes in Oxyhemoglobin Concentration in the Prefrontal Cortex and Primary Motor Cortex During Low- and Moderate- 
Intensity Exercise on a Cycle Ergometer. Adv Exp Med Biol 2017;977:241-7.

13. Cerqueira É, Marinho DA, Neiva HP, et al. Inflammatory Effects of High and Moderate Intensity Exercise-A Systematic Review. Front Physiol 2020;10:1550.

14. WHO: Global Action Plan on Physical Activity 20182030: More Active People for a Healthier World. Geneva: World Health Organization, 2018.

15. Piercy KL, Troiano RP, Ballard RM, et al. The Physical Activity Guidelines for Americans. JAMA 2018;320:2020-8.

16. Shu HF, Yang T, Yu SX, et al. Aerobic exercise for Parkinson's disease: a systematic review and metaanalysis of randomized controlled trials. PLoS One 2014;9:e100503.

17. Fiorelli CM, Ciolac EG, Simieli L, et al. Differential Acute Effect of High-Intensity Interval or Continuous Moderate Exercise on Cognition in Individuals With Parkinson's Disease. J Phys Act Health 2019;16:157-64.

18. Moore CG, Schenkman M, Kohrt WM, et al. Study in Parkinson disease of exercise (SPARX): translating highintensity exercise from animals to humans. Contemp Clin Trials 2013;36:90-8.

19. Kim Y, Lai B, Mehta T, et al. Exercise Training Guidelines for Multiple Sclerosis, Stroke, and Parkinson Disease: Rapid Review and Synthesis. Am J Phys Med Rehabil 2019;98:613-21.

20. Zoladz JA, Majerczak J, Zeligowska E, et al. Moderateintensity interval training increases serum brain-derived neurotrophic factor level and decreases inflammation in Parkinson's disease patients. J Physiol Pharmacol 2014;65:441-8.

21. Schenkman M, Moore CG, Kohrt WM, et al. Effect of High-Intensity Treadmill Exercise on Motor Symptoms in Patients With De Novo Parkinson Disease: A Phase 2 Randomized Clinical Trial. JAMA Neurol 2018;75:219-26.

22. Higgins J, Green S. Cochrane Handbook for Systematic Reviews of Interventions. London: The Cochrane Collaboration, 2009.

23. Arfa-Fatollahkhani P, Safar Cherati A, Habibi SAH, Shahidi GA, Sohrabi A, Zamani B. Effects of treadmill training on the balance, functional capacity and quality of life in Parkinson's disease: A randomized clinical trial. J Complement Integr Med 2019;17:/j/jcim.2019.17.issue-1/ jcim-2018-0245/jcim-2018-0245.xml.

24. Cugusi L, Solla P, Serpe R, et al. Effects of a Nordic Walking program on motor and non-motor symptoms, functional performance and body composition in patients with Parkinson's disease. NeuroRehabilitation
2015;37:245-54.

25. Ferraz DD, Trippo KV, Duarte GP, et al. The Effects of Functional Training, Bicycle Exercise, and Exergaming on Walking Capacity of Elderly Patients With Parkinson Disease: A Pilot Randomized Controlled Single-blinded Trial. Arch Phys Med Rehabil 2018;99:826-33.

26. Landers MR, Navalta JW, Murtishaw AS, et al. A HighIntensity Exercise Boot Camp for Persons With Parkinson Disease: A Phase II, Pragmatic, Randomized Clinical Trial of Feasibility, Safety, Signal of Efficacy, and Disease Mechanisms. J Neurol Phys Ther 2019;43:12-25.

27. Nadeau A, Pourcher E, Corbeil P. Effects of 24 wk of treadmill training on gait performance in Parkinson's disease. Med Sci Sports Exerc 2014;46:645-55.

28. van der Kolk NM, de Vries NM, Kessels RPC, et al. Effectiveness of home-based and remotely supervised aerobic exercise in Parkinson's disease: a double-blind, randomised controlled trial. Lancet Neurol 2019;18:9981008.

29. Wang J. The effect of jogging with initiation of heart rate reserve on the patients of early or middle stage. (Master Thesis) Shandong University, China, 2012.

30. Schenkman M, Hall DA, Barón AE, Schwartz RS, Mettler P, Kohrt WM. Exercise for people in early- or mid-stage Parkinson disease: a 16-month randomized controlled trial. Phys Ther 2012;92:1395-410.

31. Klamroth S, Steib S, Devan S, et al. Effects of Exercise Therapy on Postural Instability in Parkinson Disease: A Meta-analysis. J Neurol Phys Ther 2016;40:3-14.

32. Shen X, Wong-Yu IS, Mak MK. Effects of Exercise on Falls, Balance, and Gait Ability in Parkinson's Disease: A Meta-analysis. Neurorehabil Neural Repair 2016;30:512-27.

33. Cammisuli DM, Bonuccelli U, Daniele S, et al. Aerobic Exercise and Healthy Nutrition as Neuroprotective Agents for Brain Health in Patients with Parkinson's Disease: A Critical Review of the Literature. Antioxidants (Basel) 2020;9:380.

34. Uitti RJ. Treatment of Parkinson's disease: focus on quality of life issues. Parkinsonism Relat Disord 2012;18 Suppl 1:S34-6.

35. Miller Koop M, Rosenfeldt AB, Alberts JL. Mobility improves after high intensity aerobic exercise in individuals with Parkinson's disease. J Neurol Sci 2019;399:187-93.

36. Shulman LM, Katzel LI, Ivey FM, et al. Randomized clinical trial of 3 types of physical exercise for patients with Parkinson disease. JAMA Neurol 2013;70:183-90.

37. Cancela JM, Mollinedo I, Montalvo S, et al. Effects of a 
High-Intensity Progressive-Cycle Program on Quality of Life and Motor Symptomatology in a Parkinson's Disease Population: A Pilot Randomized Controlled Trial. Rejuvenation Res 2020;23:508-15.

38. Physical Activity Guidelines Advisory Committee report, 2008. To the Secretary of Health and Human Services. Part A: executive summary. Nutr Rev 2009;67:114-20.

39. Monteiro EP, Franzoni LT, Cubillos DM, et al. Effects of Nordic walking training on functional parameters in

Cite this article as: Li Y, Song H, Shen L, Wang Y. The efficacy and safety of moderate aerobic exercise for patients with Parkinson's disease: a systematic review and meta-analysis of randomized controlled trials. Ann Palliat Med 2021;10(3):26382649. doi: 10.21037/apm-20-1661
Parkinson's disease: a randomized controlled clinical trial. Scand J Med Sci Sports 2017;27:351-8.

40. Mehrholz J, Kugler J, Storch A, et al. Treadmill training for patients with Parkinson's disease. Cochrane Database Syst Rev 2015;(8):CD007830.

41. Hood RL, Liguore WA, Moore C, et al. Exercise intervention increases spontaneous locomotion but fails to attenuate dopaminergic system loss in a progressive MPTP model in aged mice. Brain Res 2016;1646:535-42. 